$65^{\text {th }}$ International Astronautical Congress, Toronto, Canada. Copyright (c) 2014 by Eleven International Publishing. All rights reserved. One or more authors of this work are employees of the government of the United States of America, which may preclude the work from being subject to copyright in United States of America, in which event no copyright is asserted in that country.

IAC-14-D3.1.8

\title{
ASTEROID REDIRECT MISSION CONCEPT: A BOLD APPROACH FOR UTILIZING SPACE RESOURCES
}

\author{
Daniel D. Mazanek \\ National Aeronautics and Space Administration (NASA), USA, Daniel.D.Mazanek@nasa.gov \\ Raymond G. Merrill \\ National Aeronautics and Space Administration (NASA), USA, Raymond.G.Merrill@nasa.gov \\ John R. Brophy \\ Jet Propulsion Laboratory - California Institute of Technology, USA, John.R.Brophy@nasa.gov \\ Robert P. Mueller \\ National Aeronautics and Space Administration (NASA), USA, Robert.P.Mueller@nasa.gov
}

The utilization of natural resources from asteroids is an idea that is older than the Space Age. The technologies are now available to transform this endeavour from an idea into reality. The Asteroid Redirect Mission (ARM) is a mission concept which includes the goal of robotically returning a small Near-Earth Asteroid (NEA) or a multi-ton boulder from a large NEA to cislunar space in the mid 2020's using an advanced Solar Electric Propulsion (SEP) vehicle and currently available technologies. The paradigm shift enabled by the ARM concept would allow in-situ resource utilization (ISRU) to be used at the human mission departure location (i.e., cislunar space) versus exclusively at the deep-space mission destination. This approach drastically reduces the barriers associated with utilizing ISRU for human deep-space missions. The successful testing of ISRU techniques and associated equipment could enable large-scale commercial ISRU operations to become a reality and enable a future space-based economy utilizing processed asteroidal materials. This paper provides an overview of the ARM concept and discusses the mission objectives, key technologies, and capabilities associated with the mission, as well as how the ARM and associated operations would benefit humanity's quest for the exploration and settlement of space.

\section{BACKGROUND}

The utilization of natural resources from asteroids is an idea that is older than the Space Age. Konstantin Tsiolkovskii included the "exploitation of asteroids" as one of his fourteen points for the conquest of space in The Exploration of Cosmic Space by Means of Reaction Motors, published in 1903. Dr. Brian O'Leary, a former NASA Group 6 astronaut candidate and Asteroidal Resources Group team leader during the 1977 NASA Ames Summer Study on Space Settlements, proposed using mass drivers to move Earth-approaching Apollo and Amor asteroids to Earth's vicinity during opportunities when the required velocity change $(\Delta \mathrm{V})$ to redirect them was low. ${ }^{1}$

Earth's closet natural location for acquiring spacebased materials is the Moon. However, the $\Delta \mathrm{V}$ necessary to access the lunar surface and the effort required to extract the low concentration of many resources in the lunar regolith (e.g., water) present significant challenges. Many Near-Earth Asteroids (NEAs) are accessible for less $\Delta \mathrm{V}$ than is required to land on and ascend from the Moon and provide valuable minerals and volatiles in concentrations much higher than found on the lunar surface.
NASA human missions to date have only utilized solar energy and planetary atmospheres (to reduce spacecraft velocity) as in-situ resources. In-situ resource utilization (ISRU) of native materials to create commodities such as propellants, consumables, and radiation shielding has only been demonstrated experimentally and with terrestrial testbeds. The incorporation of ISRU into human missions has been studied for decades, but its actual implementation has been elusive. In these studies, the resources are typically produced at the destination which introduces mission risk and complexity that needs to be reduced to make ISRU viable. Additionally, for the utilization of resources from low-gravity bodies such as asteroids, comets, and the Martian moons, the acquisition, processing, extraction and storage of beneficial compounds in these environments need to be demonstrated with sufficient efficiency to allow its inclusion in human missions.

Water is a critical resource for future human space exploration and can be used for a variety of purposes, including propellants, radiation shielding, thermal control, and human consumption, as well as a variety of non-potable applications (cleaning, plant growth, etc.). Carbonaceous asteroids are expected to contain up to 
65 ${ }^{\text {th }}$ International Astronautical Congress, Toronto, Canada. Copyright (c) 2014 by Eleven International Publishing. All rights reserved. One or more authors of this work are employees of the government of the United States of America, which may preclude the work from being subject to copyright in United States of America, in which event no copyright is asserted in that country.

$20 \%$ water by mass in the form of hydrated minerals and a similar mass percentage of other extractable volatiles (e.g., carbon dioxide, nitrogen, and carbon monoxide). Carbonaceous asteroids also have low compressive strength. This property simplifies cutting, crushing, and processing, which could be a key attribute for resource extraction and recovery. NEAs also provide plentiful oxygen from mineral oxides, metals (e.g., iron, nickel, and platinum group metals), and bulk materials that can be used for the construction of various structures, including radiation shielding.

In the process of extracting materials necessary for space exploration, such as water, significant quantities of materials returnable to terrestrial markets could be generated to help make operations profitable and sustainable. This will likely involve a commodity with a very high value-to-mass ratio along with a dramatic reduction in Earth-to-orbit launch costs and technologies to enable the cost-effective return of these materials through the atmosphere to the Earth's surface. The ability to extract materials from asteroids and return them to terrestrial markets in a profitable manner could be instrumental to opening up the space frontier for all of mankind.

\section{ARM MISSION OVERVIEW}

Following a 2011 Keck Institute for Space Studies (KISS) feasibility study ${ }^{2}$, NASA initiated a mission concept study to robotically capture an entire small NEA and redirect it to cislunar space. ${ }^{3}$ An alternative mission option also being studied by NASA would robotically capture a multi-ton boulder from the surface of a large NEA ( 100+ meter) and return it to cislunar space. ${ }^{4}$ Both approaches provide asteroidal material in an accessible location, specifically a stable Lunar Distant Retrograde Orbit (LDRO) approximately 70,000 $\mathrm{km}$ from the Moon, where astronauts can explore the asteroidal material and return samples to Earth. ${ }^{5}$ In addition, the testing of resource extraction and processing methods would be facilitated, along with the ability to gain operational experience to enable future human deep-space missions. This mission concept, known as the Asteroid Redirect Mission (ARM), leverages a high-power advanced Solar Electric Propulsion (SEP) system with high specific impulse currently in development by NASA's Space Technology Mission Directorate (STMD).

The Asteroid Redirect Mission is comprised of three main segments: 1.) Identify; 2.) Redirect; and 3.) Explore. The first segment is an observation campaign to identify and characterize candidate NEAs using ground-based facilities including optical telescopes, radar facilities, and NASA's Infrared Telescope Facility (IRTF) shown in Figures 1 and 2, and space-based assets, such as NASA's Spitzer Infrared Space Telescope and Near-Earth Object Wide-field Infrared Survey Explorer (NEOWISE) Space Telescope. The same assets are being used to help catalogue the general population of NEAs and identify those that could impact the Earth.

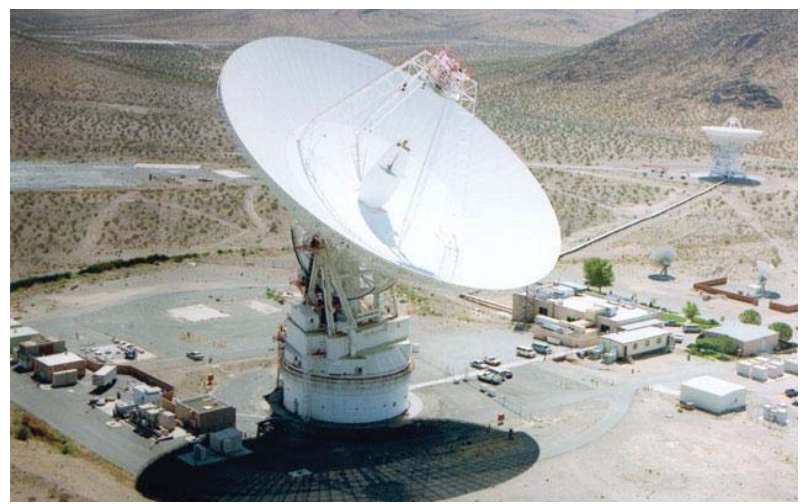

Fig. 1: 70-m dish (DSS-14) radar facility in Goldstone, California (Credit: NASA/JPL).

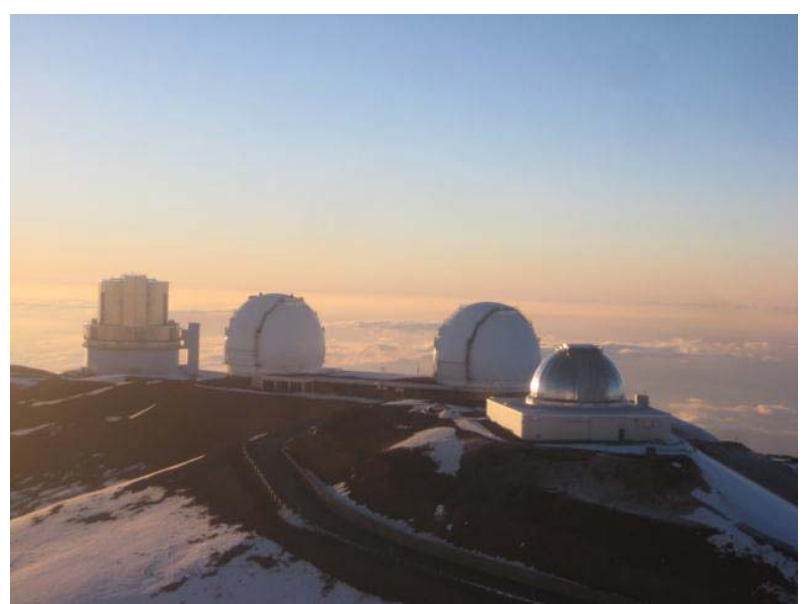

Fig. 2: Subaru, Keck, and NASA IRTF facilities on Mauna Kea's summit, shown left-to-right (Credit: NASA).

Additionally, for the robotic boulder capture option, precursor reconnaissance is planned for two candidate carbonaceous asteroids, 101955 Bennu (1999 RQ36) and $1999 \mathrm{JU}_{3}$, and one stony asteroid, 25143 Itokawa (1998 SF36), has already been visited. Bennu will be visited by NASA's Origins-Spectral InterpretationResource Identification-Security-Regolith Explorer (OSIRIS-REx) spacecraft (see Figure 3) and $1999 \mathrm{JU}_{3}$ by the Japan Aerospace Exploration Agency's (JAXA's) Hayabusa 2 spacecraft. Both will rendezvous with their respective targets in 2018 and provide detailed mapping and characterization of their physical properties. Itokawa was visited by JAXA's Hayabusa spacecraft in 2005. 
65 ${ }^{\text {th }}$ International Astronautical Congress, Toronto, Canada. Copyright (c) 2014 by Eleven International Publishing. All rights reserved. One or more authors of this work are employees of the government of the United States of America, which may preclude the work from being subject to copyright in United States of America, in which event no copyright is asserted in that country.

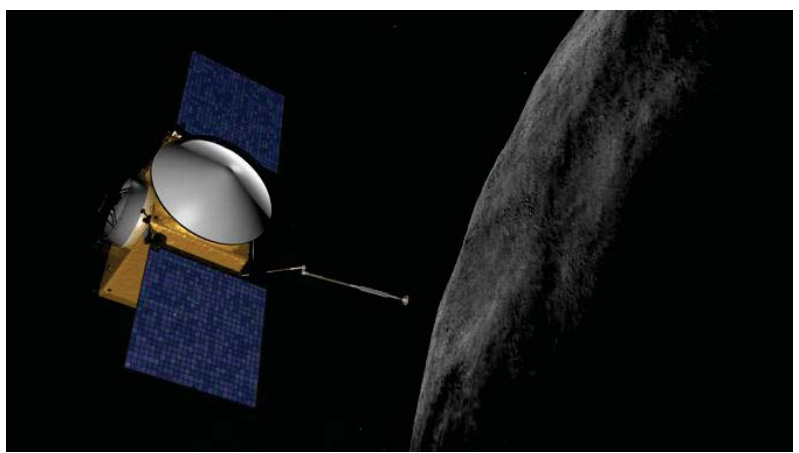

Fig. 3: OSIRIX-Rex Spacecraft (Credit: NASA).

The second segment is the Asteroid Redirect Robotic Mission (ARRM), which is the robotic portion of ARM with the goal of robotically returning a small NEA or a multi-ton boulder from a large NEA to cislunar space in the mid 2020's. The small NEA in the first option is approximately 4-10 meters in size and can have a return mass up to $\sim 1,000$ metric tons, depending on the orbit of the target NEA and the thrust-to-weight and control authority of the SEP vehicle. The returned boulder in the second option is typically 2-4 meters in size during the time period being proposed for the ARM robotic mission (launch in 2019 and return in 2025 for initial crew exploration) and the capture system is currently being designed to allow the collection of a boulder with a mass up to 70 metric tons. NEAs in very Earth-like orbits allow for the most return mass. Large NEAs with favourable orbits are less plentiful than small NEAs, but are typically easier to discover and characterize and allow boulder selection that is compatible with the permissible return mass. Capturing a small asteroid requires the mass of the entire body to be returnable. In the future, greater mass could be returned by both options as technological capability and operational experience improve along with a more complete catalogue of well-characterized NEAs. Figures 4 and 5 depict the small asteroid capture and robotic boulder capture options, respectively. For both options, the use of high-power and high specific impulse solar electric propulsion is the key enabling technology for this mission concept.

The third segment is the Asteroid Redirect Crewed Mission (ARCM) in which astronauts aboard NASA's Orion spacecraft launched on the Space Launch System (SLS) will rendezvous with the returned asteroid or boulder in a Lunar Distant Retrograde Orbit (LDRO), conduct spacewalks to collect samples, and return the samples to the Earth for analysis. The ARCM is planned for the mid 2020's.
The successful completion the Asteroid Redirect Mission would also provide many other important benefits. The following is a summary of the current objectives of the ARM:

- Conduct a human exploration mission to an asteroid in the mid-2020's, providing systems and operational experience required for human exploration of Mars.

- Demonstrate an advanced solar electric propulsion system, enabling future deep-space human and robotic exploration with applicability to the nation's public and private sector space needs.

- Enhance detection, tracking and characterization of Near Earth Asteroids, enabling an overall strategy to defend our home planet.

- Demonstrate basic planetary defense techniques that will inform impact mitigation strategies to defend our home planet.

- Pursue a target of opportunity that benefits scientific and partnership interests, expanding our knowledge of small celestial bodies and enabling the mining of asteroid resources for commercial and exploration needs.

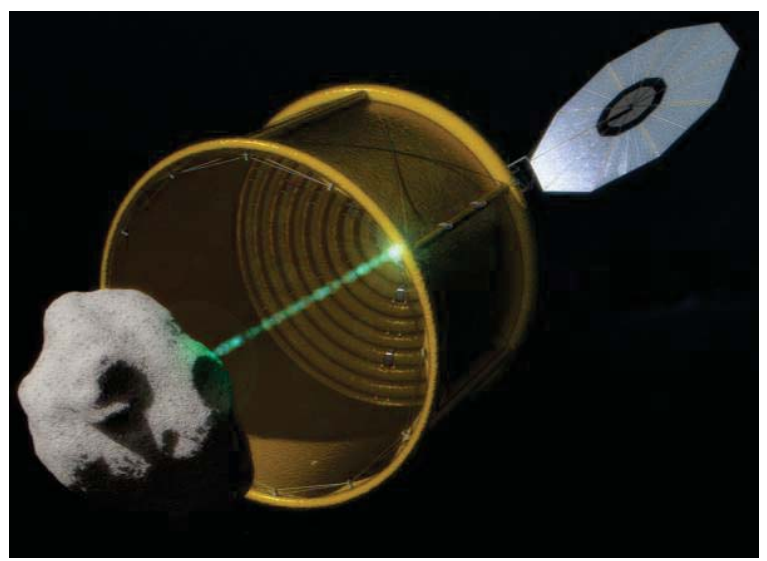

Fig. 4: ARM small asteroid capture option (Credit: NASA/JPL).

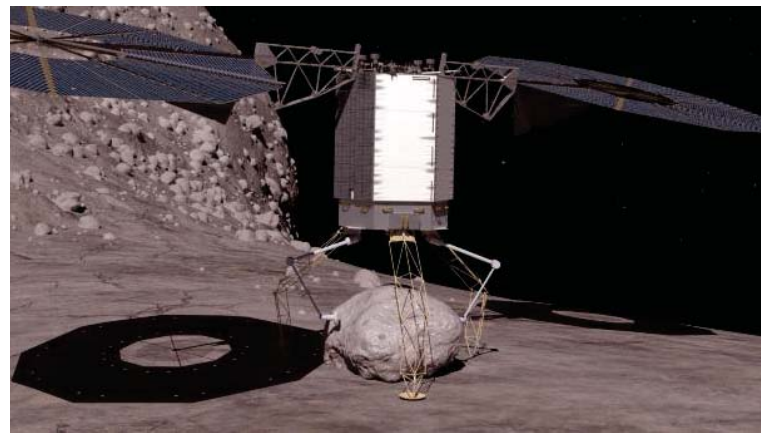

Fig. 5: ARM robotic boulder capture option (Credit: NASA/AMA, Inc.). 
65 ${ }^{\text {th }}$ International Astronautical Congress, Toronto, Canada. Copyright (c) 2014 by Eleven International Publishing. All rights reserved. One or more authors of this work are employees of the government of the United States of America, which may preclude the work from being subject to copyright in United States of America, in which event no copyright is asserted in that country.

\section{FLIGHT SYSTEM AND KEY TECHNOLOGIES AND CAPABILITIES}

The ARRM flight system, called the Asteroid Redirect Vehicle (ARV), is depicted in Figure 6 and consists of a SEP Module (SEPM), a Mission Module (MM) and a Capture Module (CM). All of the power and propulsion functions are assumed to be contained in the SEPM. The MM contains all of the spacecraft bus functions besides power and propulsion, and the Capture Module includes the unique hardware and software required for capturing the NEA or boulder. The SEPM and MM are very similar for both mission options and the CM is dependent on the mission option selected.

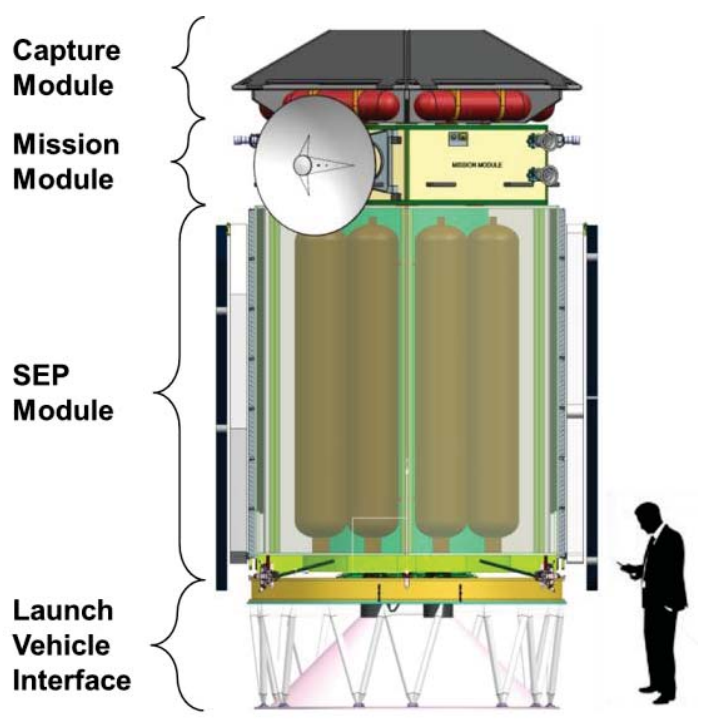

Fig. 6: Asteroid Redirect Vehicle (ARV) Modules

\section{SEP Module}

The SEPM has a unique structural design to support the xenon tanks and accommodate launch loads, but utilizes conventional thermal control and reaction control subsystems (monopropellant hydrazine).

The SEP module power subsystem provides the power to operate the electric propulsion subsystem, as well as all of the rest of the spacecraft function. A highvoltage solar array provides power to the high-voltage bus, low voltage power is created by a down-converter located in the Mission Module. A 50-kW beginning-oflife solar array (at $1 \mathrm{AU}$ ) is sized to provide $40 \mathrm{~kW}$ input to the electric propulsion system (EPS) at end-oflife, operate the non-electric propulsion loads, and charge the battery as required.

The solar array is assumed to be an advanced lightweight, flexible-blanket-type array similar to those currently under development by NASA's STMD. It is likely that either of the two SAS technologies, ROSA (upper configuration in Figure 7) or MegaFlex (lower configuration in Figure 7), if successfully developed, would be acceptable for the asteroid retrieval spacecraft. For the asteroid retrieval spacecraft the current best estimate (CBE) for the solar array specific power at 1 AU is assumed to be $145 \mathrm{~W} / \mathrm{kg}$. Each solar array wing would be mounted to a single-axis gimbal mechanism.



Fig. 7: Possible ARV Solar Array Configurations (Image Credit: NASA/JPL).

The electric propulsion system currently assumes the use of a $300 \mathrm{~V}$ Power Processing Unit (PPU) architecture and four 13.3-kW Hall thrusters also currently under development by STMD. The thrusters are configured in a 3+1 system where a maximum of three thrusters are operated simultaneously, at up to 13.3 $\mathrm{kW}$ each, and the fourth thruster is a cold spare. The thrusters operate with a specific impulse $\left(\mathrm{I}_{\mathrm{sp}}\right)$ between 1,800 and 3,000 $\mathrm{s}$ and are 50-55\% efficient depending on input power. Each thruster is capable of processing $3,300 \mathrm{~kg}$ of xenon with a low risk of wear-out failure. The SEPM also includes the xenon feed system for each thruster and 12 xenon tanks capable of holding a total of $11.1 \mathrm{t}$ of xenon (including 11\% margin). The SEPM has a unique structural design to support the xenon tanks and accommodate launch loads, but utilizes conventional thermal control and reaction control subsystems (monopropellant hydrazine). The hydrazine reaction control subsystem is used to control the tip-off rates after separation from the launch vehicle, provide three-axis control when not thrusting with the EPS, and provide for proximity operations around the NEA (small or large). It is used for capture and de-tumble for the small NEA capture option and to provide descent and ascent capability for the robotic boulder capture option.

\section{Mission Module}

The Mission Module (MM) provides the main command and data handling capability for the ARV and is responsible for ground communications and ranging, 
65 $5^{\text {th }}$ International Astronautical Congress, Toronto, Canada. Copyright (c) 2014 by Eleven International Publishing. All rights reserved. One or more authors of this work are employees of the government of the United States of America, which may preclude the work from being subject to copyright in United States of America, in which event no copyright is asserted in that country.

and control of the vehicle actuators (SEP and chemical thrusters in the SEPM and momentum wheels in the MM). The MM utilizes flight heritage avionics and provides a simple interface with the SEPM. The MM is designed within the capability of recent Jet Propulsion Laboratory (JPL) heritage based on Mars Science Laboratory (MSL) and the Soil Moisture Active Passive (SMAP) Earth satellite. It includes avionics, star trackers, MIMU, deep space transponder, reaction wheels, high-gain antenna, low-gain antennas, and thermal control hardware.

\section{Capture Module}

The Capture Module includes the sensor suite and a capture mechanism. A common sensor suite is being pursued that can facilitate automated rendezvous and docking/capture (AR\&D) sensors for both the robotic and crewed segments of the ARM and eliminate the cost of multiple sensor developments and qualification programs. The proposed sensor suite currently consists of one or more visible wavelength cameras, a threedimensional Lidar, and a long-wavelength infrared camera for robustness and situational awareness. ${ }^{6}$

The main difference in the ARV results from the mission option selected for the ARRM. For the small asteroid capture option, the CM is designed to encapsulate the entire small NEA to accommodate the possibility that the object could be a "rubble pile" comprised of various size particles held together by inter-particle forces and the NEA's weak gravitational field. For this option, the capture mechanism is a large deployable structure that enables capture of an entire, small NEA, with a mass of up to $\sim 1,000 \mathrm{t}$ and a rotation period of greater than 2 minutes, inside a high-strength bag. The asteroid is captured by having the spacecraft match the spin state of the object, positioning the asteroid inside the open bag, and then closing the bag around the asteroid. Cinch lines pull the bag closed and hold the spacecraft against the asteroid. The capture mechanism will be jointly development with industry. This approach is capable of handling the likely extremes of possible NEA properties ranging from a weak rubble pile to monolithic rock.

For the robotic boulder capture option, the Capture Module provides all of the functions necessary to perform the following key functions: 1) asteroid and boulder characterization; 2) onboard asteroid- and boulder-relative navigation; 3) asteroid surface interaction; 4) boulder capture; 5) boulder restraint during the return flight; and 6) enhanced support of crew extra-vehicular activity (EVA), including robotic preparation of the work site prior to crew arrival and between EVAs, and potential robotic collection and caching of boulder surface and sub-surface samples for crew evaluation, collection, and return to Earth. ${ }^{7}$
Current mission refinement efforts are focused on an option that includes a Robot Subsystem and a Contact and Restraint Subsystem (CRS). The Robot Subsystem consists of two 7-DOF arms currently under development at NASA's Satellite Servicing Capabilities Office (SSCO) for use on proposed satellite servicing missions. The boulder capture end-effector tool is the JPL Microspine Gripper, which uses hundreds of fishhook-like spines to opportunistically grab the surface features of natural objects. The CRS arrests the descent of the ARV and stabilizes it on the surface of the asteroid, as well as providing the means of departing the surface using a mechanical push-off. This approach has been investigated to avoid directly pluming the surface of the NEA with the RCS so as to minimize contaminating the spacecraft's solar arrays and other sensitive components with dust or larger particles. The CRS design consists of a set of three Spaceframe arms, each with three or four degrees of freedom and contact pads at their tips. The contact pads accommodate geological/geographical context sample collectors to accompany the captured boulder.

\section{PROCESSING OF ASTEROIDAL MATERIAL}

There are three main anticipated types of asteroidal materials for ISRU:

1. Regolith

a. Oxygen from mineral oxides

b. Iron, nickel, \& platinum group metals

2. Water and other volatiles bound in regolith

3. Bulk material for radiation shielding \& construction

These materials are not ubiquitous and the large variety of asteroids means that an assay must first be performed to determine which asteroids are desirable targets of opportunity for ISRU purposes. This assay is currently in progress, and uses reflectance and spectral data to determine asteroid composition. However, data obtained directly from NEAs is limited, so for the purposes of this paper, only the taxonomy of collected meteorites from asteroids will be considered. Even though this is a highly biased population, the mineralogical composition has been precisely measured and therefore provides the best source of information for asteroid ISRU analysis and mission planning.

The stony asteroids are dominated by silicates and therefore are good candidates for oxygen extraction via chemical reduction processes. Ninety six percent of meteorites from asteroids belong to this category, ${ }^{8}$ and these consist of chondrites (88\%), which are primitive, undifferentiated and contain $30-100 \%$ oxidized iron, and achondrites (8\%) which are silicate rich igneous material containing 99\% silicates and oxides. 
65 $5^{\text {th }}$ International Astronautical Congress, Toronto, Canada. Copyright (c) 2014 by Eleven International Publishing. All rights reserved. One or more authors of this work are employees of the government of the United States of America, which may preclude the work from being subject to copyright in United States of America, in which event no copyright is asserted in that country.

The remaining meteorites consist of Stony Iron asteroids (1\%) and Iron (M) asteroids (3\%). The Stony Irons most likely consist of $50 \%$ ferrous metal alloys and $50 \%$ silicates (mostly olivine), while the Irons consist of $99 \%$ metallic Fe-Ni-Co alloys based on recovered meteoritic evidence on Earth. ${ }^{9}$

For ISRU, the metallic materials will be very valuable for making parts via various manufacturing processes, both traditional (casting, machining, forging, etc.), as well as new additive manufacturing techniques such as Selective Laser Sintering (SLS) and Selective Laser Melting (SLM). These parts will initially be used as spares and eventually could form the basis of a solar system industry where only digital information is transmitted form Earth, while the materials are locally sourced in space. ${ }^{10}$

The chondrite meteorites can be further subclassified into ordinary chondrites, (H, L, LL), carbonaceous chondrites, (C-Type: CI, CM, CO, CV) and enstatite chondrites, (Eh, EL). The Ordinary chondrites contain large quantities of oxidized iron and nickel, so they will also be a good source of metals for manufacturing with $\mathrm{O}_{2}$ as an additional valuable resultant resource. The enstatite chondrites contain 60$80 \%$ silicates and therefore will also be a valuable source of $\mathrm{O}_{2}$. Since chemical $\mathrm{H}_{2} / \mathrm{O}_{2}$ rocket propulsion has a high specific impulse $\left(\mathrm{I}_{\mathrm{sp}}\right)$ in vacuum of approximately 450 seconds and the oxidizer typically comprises over $80 \%$ of the total propellant mass, $\mathrm{O}_{2}$ is an extremely valuable commodity for in-space transportation using high-thrust, chemical propulsion.

In order to acquire both the fuel and the oxidizer for a $\mathrm{H}_{2} / \mathrm{O}_{2}$ rocket propulsion system at an asteroid, the most useful target bodies will be carbonaceous chondrites (CI and CM types in particular). These are highly oxidized with little or no free metal and also contain relatively abundant volatiles: up to $20 \%$ bound water and $6 \%$ organic material. ${ }^{8}$ The water can be electrolyzed to create $\mathrm{H}_{2} / \mathrm{O}_{2}$ propellants and the organic material can be used to create other useful hydrocarbons, including possibly making $\mathrm{CH}_{4}$ fuel which is easier to cryogenically store and condition than the lower temperature and less dense $\mathrm{H}_{2}$

D-type and P-type asteroids are believed to have a composition of organic-rich silicates, carbon and anhydrous silicates, possibly with water ice in their interiors. The expected resources available from different asteroid types are summarized in Table 1.,11

\section{Elimination of Long NEA Communications Delays}

Long communications delays (many minutes each way) preclude the direct telerobotic operation of ISRU equipment at NEA's and full robotic autonomy is not sufficiently advanced to execute a complex resource extraction mission independently. Fortunately, bringing material to cislunar space allows processing of materials by humans without the Earth communications delays associated with NEAs in their native orbits, and without the long travel times from Earth for the crew. The ARV's ability to transport the asteroidal material to the LDRO allows for processing in cislunar space where the space faring capabilities will exist.

\begin{tabular}{|c|c|c|}
\hline Asteroid Type & $\begin{array}{c}\text { Description } \\
\text { (major surface } \\
\text { minerals) }\end{array}$ & $\begin{array}{l}\text { Expected } \\
\text { Resources }\end{array}$ \\
\hline $\begin{array}{c}\text { C } \\
\text { (carbonaceous: } \\
\text { CI, CM, CO, } \\
\text { CV) }\end{array}$ & clays, organics & $\begin{array}{l}\text { water (hydrated } \\
\text { minerals): } \\
\qquad 10-20 \% \\
\text { simple } \\
\text { hydrocarbons, } \\
\text { metals }\end{array}$ \\
\hline $\mathrm{D}, \mathrm{P}$ & $\begin{array}{l}\text { organics, } \\
\text { anhydrous } \\
\text { silicates (?) }\end{array}$ & volatiles, water \\
\hline M (metal) & metals, enstatite & $\begin{array}{l}\text { Fe, Ni, Pt- } \\
\text { group metals }\end{array}$ \\
\hline S (stony) & $\begin{array}{c}\text { olivine, } \\
\text { pyroxene, metals }\end{array}$ & $\begin{array}{c}\text { oxides, } \\
\text { oxygen, metals }\end{array}$ \\
\hline
\end{tabular}

Table 1. Expected resources of value in various asteroid types.

\section{ISRU Processing Techniques - Mining Cycle}

The physical state and accessibility of the resources are very important to establish the viability of mining them. Most asteroids (other than metallic) are thought to be low-strength rubble piles, The regolith on asteroids has a much higher thermal inertia, and much lower gravity than on Moon. We can expect courser soils, and more boulders. Micro-impacts and regolith gardening can result in size segregation. The solar wind may deplete the smallest size fraction and the larger materials may be preferentially retained on the surface of the asteroid. In addition, fine materials may be retained at depth in the soil profile. These factors must all be taken into account and the resource must be acquired, size sorted, beneficiated, processed and the tailings disposed of. Table 2 summarizes some relevant ISRU methods for extracting resources. ${ }^{9}$

\section{Potential ISRU Products}

Based on the evidence provided by meteorites, it is likely that the initial uses of resources in human space exploration will be for rocket propellants, life support fluids, structural metals, refractory heat shields and radiation shielding. ${ }^{8}$

The most effective radiation shielding to protect the crew from solar particle events (SPEs) and Galactic Cosmic Rays (GCRs) are, in general, low atomic mass elements. Since hydrogen has the lowest atomic mass, it 
65 ${ }^{\text {th }}$ International Astronautical Congress, Toronto, Canada. Copyright (C) 2014 by Eleven International Publishing. All rights reserved. One or more authors of this work are employees of the government of the United States of America, which may preclude the work from being subject to copyright in United States of America, in which event no copyright is asserted in that country.

\begin{tabular}{|c|c|}
\hline Possible Available Resources & Methods to Extract Resources \\
\hline $\begin{array}{l}\text { Volatiles (simple compounds of hydrogen, } \\
\text { oxygen, carbon, sulfur and nitrogen) Rarely } \\
\text { nitrogen, halogens and noble gases. }\end{array}$ & Heating: Microwave or Solar Thermal processes \\
\hline Water - primarily as hydrated minerals & $\begin{array}{l}\text { A. Heating: Microwave or Solar Thermal processes } \\
\text { B. Ionic Liquid Acid dissolution, } \\
\text { C. } \mathrm{H}_{2} \mathrm{SO} 4 \text { or } \mathrm{HF} \text { dissolution }\end{array}$ \\
\hline Metal Oxides (for oxygen) & $\begin{array}{l}\text { A. Ionic Liquid Acid dissolution } \\
\text { B. } \mathrm{H}_{2} \mathrm{SO} 4 \text { or } \mathrm{HF} \text { dissolution } \\
\text { C. Hydrogen Reduction } \\
\text { D. Carbothermal Reduction } \\
\text { E. Molten Oxide Electrolysis }\end{array}$ \\
\hline Metal Oxides (for metal) & $\begin{array}{l}\text { A. Molten Oxide Electrolysis } \\
\text { B. Ionic Liquid dissolution followed by electrolysis }\end{array}$ \\
\hline Elemental Metals & $\begin{array}{l}\text { A. Heating: Microwave or Solar Thermal processes } \\
\text { B. Molten Oxide Electrolysis } \\
\text { C. Ionic Liquid dissolution followed by electrolysis }\end{array}$ \\
\hline Platinum Group Metals & $\begin{array}{l}\text { A. Heating: Microwave or Solar Thermal processes } \\
\text { B. Molten Oxide Electrolysis } \\
\text { C. Ionic Liquid dissolution followed by electrolysis }\end{array}$ \\
\hline Metal Oxides (oxygen and metals) & $\begin{array}{l}\text { A. Molten Oxide Electrolysis } \\
\text { B. Ionic Liquid dissolution followed by electrolysis }\end{array}$ \\
\hline Elemental Metals (Primarily Iron and Nickel) & $\begin{array}{l}\text { A. Heating: Microwave or Solar Thermal processes } \\
\text { B. Molten Oxide Electrolysis } \\
\text { C. Ionic Liquid dissolution followed by electrolysis }\end{array}$ \\
\hline Platinum Group Metals & $\begin{array}{l}\text { A. Heating: Microwave or Solar Thermal processes } \\
\text { B. Molten Oxide Electrolysis } \\
\text { C. Ionic Liquid dissolution followed by electrolysis }\end{array}$ \\
\hline
\end{tabular}

Table 2. Potential extraction methods for demonstration based on desired resources.

is the most desirable material for ISRU shielding. Secondary radiation effects complicate the situation and must also be accounted for in the shielding design. CI \& CM chondrites are about $30-40 \%$ less dense (fewer high atomic mass minerals), rich in water and $\mathrm{OH}$, and by far provide the best shielding material. All other stony meteorites are about the same and less effective than water-rich carbonaceous chondrites. ${ }^{9}$ Radiation shields can also be made from the bulk regolith and boulders on an asteroid. If enough regolith/boulders (several meters thick) are used in the form of structures, sand bags, panels, or other bulk mass, then effective radiation protection can be achieved for humans.

Refractory heat shields made from silicates have a high potential to provide ablative heat shield protection during planetary atmosphere entries. ${ }^{12}$ These heat shields could be made at a returned asteroid using advanced in-space regolith manufacturing methods and then used for Mars aero-capture or to return resources to the Earth's surface. Initial materials tests have shown promise but the overall concept is at a very low technology readiness level (TRL 3).

\section{IMPACT OF ASTEROIDAL RESOURCES}

Asteroids (main belt and near-Earth) represent a vast source of resources that reside outside of deep gravity wells. With the development of extraction, processing, storage and transfer technologies, bulk materials can be leveraged for fuel, mission consumables, and radiation shielding. 3-D printing technologies enable in-situ manufacturing for structures, spacecraft components, spare parts and even rocket engines.

Recent developments in electric propulsion technologies, such as NASA's Deep Space 1 and Dawn spacecraft, have opened the door to potential prospecting missions and the ability to return large amounts of asteroidal material to the Earth-Moon system as proposed for the ARM. With these large samples, ISRU technologies can be developed and matured quickly by leveraging the cislunar transportation systems that will become available in the coming decade and can be developed on an industrial scale. As the reliability of the ISRU equipment and operations increases, the robots and machines can also be sent to asteroids to process them in their native orbits. Depending on the application, it may be more efficient to process fuel and other feedstocks at their source since some of the processed fuel can be used to return the remainder to the point of use. Conversely, if a large enough percentage of the material can be converted into useful forms, it may be more practical to bring the asteroidal material to cislunar space for processing. Ultimately, both modes of processing 
65 $5^{\text {th }}$ International Astronautical Congress, Toronto, Canada. Copyright (c) 2014 by Eleven International Publishing. All rights reserved. One or more authors of this work are employees of the government of the United States of America, which may preclude the work from being subject to copyright in United States of America, in which event no copyright is asserted in that country.

asteroidal resources may be implemented in the future. Resources for use in the Earth-Moon system would likely come from Near-Earth Asteroids and resources for use in the Mars system would be processed from Phobos, Deimos, or Near-Mars Asteroids. In this way resources from asteroids can be used at the point of departure from Earth and eventually at the destination once the systems are reliable enough for human missions. Eventually, main belt asteroids could be harvested. However, the farther from the sun a resource is, the longer it takes to reach it and return from it on minimum propellant trajectories.

\section{NASA Missions}

It is NASA's role to advance the development of technologies and requisite systems to prove their feasibility and transfer that technology to industry for large-scale development. By developing long-lead technologies and providing an initial market for asteroidal resources in space, a new trans-lunar economy can be born. In the spirit of exploration, NASA has conceptualized or planned future human missions to the lunar surface, NEAs, the Martian moons, the surface of Mars, and even the outer planets Jupiter and Saturn. In a future where the Earth-Moon system is accessible by commercial launch providers and space-based propellants are readily available for use, launch mass requirements can be greatly reduced since propellant for any of these pioneering and exploration missions is a large portion of the necessary mission mass. With the development of a reusable stage that is fuelled by the resources of asteroids it is conceivable to travel to the surface of the Moon or Mars by only launching the payload elements to Low-Earth Orbit (LEO) and using ISRU-derived resources for the remainder of the trip. In a manner analogous to oceangoing vessels circumnavigating the Earth, travellers can rest in ports and acquire fuel and supplies before embarking on the next leg of their journey.

The successful processing of asteroidal materials would provide equipment and operational techniques that could be used in future processing of in-situ materials derived from the Martian moons, which may contain hydrated minerals and possibly water ice. If the extraction of water ice from Martian regolith is performed on the surface, in addition to or instead of atmospheric processing, the lessons learned from processing asteroidal materials could provide valuable experience for ISRU operations. This could also be true for the processing of lunar regolith. Regardless of whether or not the equipment and operations needed to process asteroidal materials is directly extensible to ISRU at other destinations, the simple act of learning to embrace the use of in-situ resources would provide a fundamental shift in the formulation and implementation of future NASA human missions.

\section{Commercial Entities}

Commercial entities exist today with the stated goal of developing space resources. Planetary Resources, Inc. and Deep Space Industries were created recently to prospect for and eventually exploit asteroidal resources for use both in space and in terrestrial markets. By providing material in an accessible location, those companies and others will be better able to demonstrate ISRU techniques and begin maturing their resource extraction, processing, transfer, and storage capabilities while they also develop the systems to deliver them to NEAs in their native orbits. By enabling commercial entities to control part or all of the resource extraction, transport, and storage operations, industrial expansion in space is not limited to supporting exploration only. This also allows the terrestrial mining and manufacturing industries to more easily leverage lower cost space transportation and other required assets. For example, rather than sending new components from Earth to operate in the lunar vicinity, they can be designed and tested on Earth, and then manufactured in-situ with local resources utilized for the bulk of the system mass and volume. The presence of asteroidal material in cislunar space could advance commercial mining efforts and the availability of ISRU products by up to a decade. $^{13}$

\section{Lunar Surface Access}

In a manner similar to cargo delivery from LEO to lunar orbit with propellant produced from asteroidal material as discussed earlier, lunar surface access can be enabled by use of propellant extracted from asteroids. A re-usable single stage lunar lander with minimal crew accommodations could deliver crew or cargo to the Moon. These cargo deliveries coupled with the capability to conduct in-situ manufacturing of the infrastructure needed on the surface of the Moon could enable a bootstrapped industrial colony on the moon that requires a minimal amount of Earth-based resources once it is operation.

In the not-too-distant future, a combination of material returned from NEAs and lunar resources could provide the materials and mass required to construct a lunar space elevator. This innovation could be a key capability to facilitate the permanent settlement of the Moon.

\section{Mars Cyclers}

A crewed trip to Mars presents significant challenges to life that flourishes in a 1-g, radiation shielded environment like we have on Earth. The SEP technology that is being proposed to return asteroidal 
65 $5^{\text {th }}$ International Astronautical Congress, Toronto, Canada. Copyright (c) 2014 by Eleven International Publishing. All rights reserved. One or more authors of this work are employees of the government of the United States of America, which may preclude the work from being subject to copyright in United States of America, in which event no copyright is asserted in that country.

material could also be used to re-direct an asteroid onto a ballistic two-synodic period Earth-Mars Cycling trajectory over a period of approximately ten years. Two of these cyclers would be required for crewed access to Mars every other departure opportunity (four cyclers for every opportunity). These cyclers could serve as an "oasis" in space for humans to hyperbolically rendezvous with and ride to and from the Mars system. These asteroids could initially be processed for resources and industrial robotics could transform them into radiation shielded transfer vehicles. Eventually, they could be manipulated to spin at a sufficient rate to provide a partial-g to 1-g artificial gravity environment.

Access to Main Asteroid Belt and Beyond

Most of asteroidal material in our solar system resides in the main belt between the orbits of Mars and Jupiter. Ceres is the largest of the main belt asteroids and the first to be discovered on January 1, 1801 by the Italian astronomer Giuseppe Piazzi. Ceres was reclassified as a dwarf planet by the International Astronomical Union (IAU) in 2006. The surface composition of Ceres is similar to that of C-type asteroids. ${ }^{14}$ In January of 2014, water vapor emissions were detected from several mid-latitude sources on Ceres by the Herschel Space Observatory. ${ }^{15}$ NASA's Dawn spacecraft is scheduled to arrive at Ceres in early 2015 and begin a five month reconnaissance of this potential source of in-situ resources. With a surface gravity of only approximately $17 \%$ of the Moon, Ceres may provide an abundant supply of accessible resources for exploration in the outer regions of our solar system.

As mankind ventures further and further into space, the need for in-situ resources will inevitably continue. The Kuiper belt and Oort cloud are believed to contain vast numbers of icy bodies that could one day be harvested for their resources. The Kuiper belt exists past the orbit of Nepture and the hypothesized Oort cloud may extend a quarter of the way to the Sun's nearest neighbour, Proxima Centauri. Whether or not it turns out to be practical to utilize these resources in the future exploration and settlement of space remains to be seen, but their presence provides the inspiration that more distant resources are available that could one day help humans to span the gulf of interstellar space to settle on an Earth-like planet orbiting a star in another solar system.

\section{SUMMARY}

The technologies are now available to transform the concept of redirecting large amounts of asteroidal material and the extraction of the resources contained within from an idea into reality. SEP is the key technology required to efficiently transport mass between interplanetary locations. Additionally, the power available when the SEP system is not operating could be used to power resource extraction and processing equipment.

The ARM changes the ISRU paradigm by delivering tons to hundreds of tons of asteroidal material to cislunar space where ISRU techniques can be demonstrated and refined. At this location, these demonstrations can be performed telerobotically from Earth with very little latency, and the associated equipment can be serviced by astronauts when needed. Ultimately, the paradigm shift enabled by the ARM concept would allow in-situ resources to be used at the human mission departure location (i.e., cislunar space) versus exclusively at the deep-space mission destination. This approach could drastically reduce the barriers associated with utilizing ISRU for human deepspace missions by allowing the systems to be fully tested and checked out prior to crew departure. By providing operational confidence of how the ISRU products will perform in conjunction with the vehicles and crew members, this approach would significantly reduce the mission risk associated with ISRU and also increase the likelihood that ISRU can be successfully implemented at deep-space locations.

Determining the composition of the NEA is critical. Returning the right type of asteroidal material to cislunar space would allow ISRU demonstrations to be conducted to determine how successfully the bulk asteroidal material may be converted into useful products. The testing and validation of ISRU equipment and methods could enable large-scale commercial ISRU operations to become a reality. The ARM goal of returning many tons of water-rich carbonaceous material to cislunar space could be instrumental in enabling a future space-based economy utilizing processed asteroidal materials. This, in turn, will aid humanity's expansion into the solar system, and possibly interstellar journeys in the not too distant future, by tapping the vast resources available from asteroids. 
$65^{\text {th }}$ International Astronautical Congress, Toronto, Canada. Copyright (c) 2014 by Eleven International Publishing. All rights reserved. One or more authors of this work are employees of the government of the United States of America, which may preclude the work from being subject to copyright in United States of America, in which event no copyright is asserted in that country.

\section{References}

${ }^{1}$ O’Leary, B. T., Mass Driver Retrieval of Earth-Approaching Asteroids, AIAA Paper 77-528, 1977.

${ }^{2}$ Brophy, J., Friedman, L., Culick, F., et al., Asteroid Retrieval Feasibility Study, Keck Institute for Space Studies, California Institute of Technology, Jet Propulsion Laboratory, Pasadena, California, April 2, 2012.

${ }^{3}$ Strange, N., et al., "Overview of Mission Design for NASA Asteroid Redirect Robotic Mission Concept," 33rd International Electric Propulsion Conference, The George Washington University, Washington, D.C., October 2013.

${ }^{4}$ Mazanek, D. D., Merrill, R. G., Belbin, S. P., Reeves, D. M., Earle, K. D., Naasz, B. J., and Abell, P. A., "Asteroid Redirect Robotic Mission: Robotic Boulder Capture Option Overview," AIAA SPACE 2014 Conference and Exposition, San Diego, CA, August 2014.

${ }^{5}$ Stich, S., "Asteroid Redirect Crewed Mission Building Human Exploration Capabilities," $11^{\text {th }}$ Meeting of the NASA Small Bodies Assessment Group, Washington, D.C., July 2014, Lunar and Planetary Society, http://www.lpi.usra.edu/sbag/meetings/jul2014/presentations/1030_Wed_Stitch_ARM_Crewed_Mission.pdf. [cited 02 September 2014].

${ }^{6}$ McDonald, M. A., et al., "Extensibility of Human Asteroid Mission to Mars and Other Destinations," AIAA SpaceOps 2014 13th International Conference on Space Operations, May 2014.

${ }^{7}$ Belbin, S. P., and Merrill, R. G., "Boulder Capture System Design Options for the Asteroid Robotic Redirect Mission Alternate Approach Trade Study," AIAA SPACE 2014 Conference and Exposition, San Diego, CA, August 2014.

${ }^{8}$ Lewis, J. S., and Hutson, M. L., “Asteroidal Resource Opportunities Suggested by Meteorite Data,” Resources of Near-Earth Space, 523-542, 1993.

${ }^{9}$ Chabot, N., et al., "Report of the Small Bodies Assessment Group Asteroid Redirect Mission

Special Action Team - DRAFT" Lunar and Planetary Institute, Small Bodies Assessment Group Website, http://www.lpi.usra.edu/sbag/documents/SBAG_ARM_SAT_07_30_14.pdf. [cited 02 September 2014].

${ }^{10}$ Metzger, P. T., Muscatello, A., Mueller, R. P., \& Mantovani, J., “Affordable, Rapid Bootstrapping of the Space Industry and Solar System Civilization,” Journal of Aerospace Engineering, 26(1), pp. 18-29 (2012).

${ }^{11}$ Britt, D. T., Guy Consolmagno, S. J., and Lebofsky, L., "Chapter 26: Main-Belt Asteroids," Encyclopedia of the Solar System, Eds. Spohn,T., Breuer, D., and Johnson, T., Elsevier, 583-601 (2014).

${ }^{12}$ Hogue, M. D., Mueller, R. P., Sibille, L., Hintze, P. E., \& Rasky, D. J., "Regolith-Derived Heat Shield for Planetary Body Entry and Descent System with In-Situ Fabrication," Proceedings of the ASCE Earth \& Space Conference, pp. 526-536 (2012).

${ }^{13}$ Lewicki, C. (President and Chief Engineer of Planetary Resource Inc.), private communication at NASA Blue Sky meeting March 24-24, 2014.

${ }^{14}$ Rivkin, A.S., Volquardsen, E.L., and Clar, B.E., "The Surface composition of Ceres: Discovery of carbonates and iron-rich clays," Icarus, 185:563-567 (2006).

${ }^{15}$ Küppers, M., et al., "Localized sources of water vapour on the dwarf planet (1) Ceres," Nature, 505 (7484): 525-527 (2014). 